\title{
Downregulation of SNAIL sensitizes hepatocellular carcinoma cells to TRAIL-induced apoptosis by regulating the NF- $\mathrm{NB}$ pathway
}

\author{
ZHAOJUN WAN $^{1 *}$, HUAZHENG PAN $^{2 *}$, SHIHAI LIU ${ }^{3}$, JINGJUAN ZHU ${ }^{1}$, \\ WEIWEI QI ${ }^{1}, \mathrm{KAI} \mathrm{FU}^{1}$, TENG ZHAO ${ }^{1}$ and JUN LIANG ${ }^{1}$ \\ Departments of ${ }^{1}$ Oncology and ${ }^{2}$ Clinical Laboratory, ${ }^{3}$ Central Laboratory, The Affiliated \\ Hospital of Qingdao University, Qingdao, Shandong 266003, P.R. China
}

Received September 23, 2014; Accepted January 9, 2015

DOI: 10.3892/or.2015.3743

\begin{abstract}
Hepatocellular carcinoma (HCC) is the sixth most common cancer and the second most lethal cancer worldwide. Evidence has shown HCC cell resistance to TRAIL-mediated apoptosis. In a previous study, we verified that silencing SNAIL downregulated the growth of HCC cells. In addition, the mechanism of resistance to TRAIL in HCC cells was connected with the activation of nuclear factor- $\kappa \mathrm{B}(\mathrm{NF}-\kappa \mathrm{B})$. Thus, it was hypothesized that the downregultaion of SNAIL sensitizes HCC cells to TRAIL-induced apoptosis by regulating the $\mathrm{NF}-\kappa \mathrm{B}$ pathway. In the present study, the most effective lentiviral vectors carrying shRNA against SNAIL were selected and adenoviral vectors harboring TRAIL were constructed. The expression of SNAIL and TRAIL was detected by quantitative PCR and western blotting. HCC cell viability and apoptosis were assessed using an MTT assay and the Hoechst test. To determine how to sensitize HCC cells to TRAIL-induced apoptosis after silencing SNAIL, p53 was assessed by western blot analysis. We also investigated the expression of Bcl-xL, cIAP2, survivin and Raf-1 protein using western blot analysis and the apoptotic degree of HuH-7 cells was detected using the Hoechst test following the suppression of each gene, which was a possible molecular mechanism to sensitive TRAIL-induced apoptosis through the downregulation of SNAIL in HCC cells. Silencing SNAIL resulted in increased apoptosis by enhancing sensitization to TRAIL in all the HCC cells. Additionally, p53 protein was upregulated in HuH-7 cells. Expression of Bcl-xL, cIAP2, survivin and
\end{abstract}

Correspondence to: Professor Jun Liang, Department of Oncology, The Affiliated Hospital of Qingdao University, 16 Jiangsu Road, Qingdao, Shandong 266003, P.R. China

E-mail: liangjun1959@aliyun.com

${ }^{*}$ Contributed equally

Key words: hepatocellular carcinoma, SNAIL, TRAIL, lentivirus, adenovirus, NF- $\mathrm{KB}$
Raf-1 was downregulated following silencing of SNAIL, while downregulation of any of the proteins contributed to SNAIL suppression enhancing HCC cell sensitivity to TRAIL-induced apoptosis, with the exception of cIAP2. The results demonstrated that silencing SNAIL can sensitize TRAIL-induced apoptosis in $\mathrm{HCC}$ cells by upregulating p53 protein and by regulating related genes of the $\mathrm{NF}-\kappa \mathrm{B}$ pathway such as Bcl-xL, survivin and Raf-1.

\section{Introduction}

Hepatocellular carcinoma (HCC), as the most common type of primary liver cancer, is the sixth most common cancer globally, with a prevalence of $\sim 600,000$ individuals worldwide. $\mathrm{HCC}$ is the second most lethal cancer worldwide and one of the most rapidly growing cancer types in Asia, particularly in China $(1,2)$. Reaction of biochemistry and gene control of apoptosis are important in hepatocarcinogenesis. Failure of apoptosis further promotes the development of cancer, owing to tumor occurrence. The majority of HCC cells are markedly resistant to the stimuli of inducing apoptosis, which can lead to HCC cell anti-apoptosis (3). Many factors prevent HCC cells from apoptosis, including anti-apoptotic members Bcl-2/xL (4), survivin and cyclooxygenase (COX)-2 (5,6). The abnormal expression of these factors contributes to resistance to HCC cell apoptosis, resulting in loss of tumor control and patient death. Therefore, identification of a new mechanism to convert resistance to apoptosis and promote apoptosis for the treatment of HCC is crucial.

TRAIL (TNF-related apoptosis-inducing ligand), also known as Apo2 ligand (Apo2L), is a member of the TNF family, and has been shown to induce cell apoptosis in various types of tumor but not normal cells (7). Five receptors, including TRAIL-R1/DR4, TRAIL-R2/DR5, TRAIL-R3/DcR1, TRAIL-R4/DcR2 and TNFR-homologue osteoprotegerin (OPG) have been identified thus far (8). However, of these receptors, only TRAIL-R1/DR4 and TRAIL-R2/DR5 induce apoptosis $(8,9)$. Several tumor cell lines exhibiting resistance to TRAIL-mediated apoptosis include HCC cell lines $(3,10,11)$. However, the mechanism involved in the resistance to TRAIL-induced apoptosis in HCC cells is mainly connected 
with activation of the NF- $\mathrm{B}$ pathway, upregulation of apoptotic inhibitors, such as cFLIP, IAP and anti-apoptotic molecules, such as certain Bcl-family members (12-15). TRAIL is involved in inhibition of cancer. Thus, the upregulation of HCC cell sensitization in TRAIL-induced apoptosis by mediating the above targets is significant in HCC treatment.

SNAIL, as a transcriptional repressor, has a structure of zinc finger $(\mathrm{ZF})$ which plays an important role in physiological processes such as embryonic development, and in a wide variety of pathologic processes $(16,17)$. SNAIL has been found to enhance cancer invasion and metastasis in various malignancies $(16,18,19)$. Similarly, SNAIL is crucial to the pathological progression of HCC. Evidence suggests that SNAIL contributed to tumor progression by inducing EMT to transform epithelial cells into mesenchymal ones (18). In addition, a study showed that SNAIL was induced and accelerated cell activity by repressing E-cadherin expression and upregulating MMP expression in HCC both in vitro or in vivo (19). Findings of another study showed that knockdown of SNAIL reduced proliferation and viability of HCC cells by increasing the expression of E-cadherin (20). All the aforementioned mechanisms suggested that SNAIL affects the viability and migration of HCC cells. Nevertheless, whether SNAIL affects the apoptosis of HCC cells remains to be determined. Thus, it is imperative to examine the characteristics of SNAIL with regard to the occurrence and progression of $\mathrm{HCC}$, particularly in cell apoptosis.

RNA interference (RNAi) has emerged as a new and an indispensable tool for loss of gene function in eukaryotes in order for small-interfering RNA (siRNA), a class of synthetic short double-stranded RNA, to emerges at the appopriate time point $(21,22)$. siRNA functions by inducing the silencing of gene by guiding endonucleolytic cleavage of message RNA (mRNA) or repressing its translation $(23,24)$, while siRNA cannot maintain long-term silencing of the interfering gene. Thus, to induce the gene to stable and reversible silencing for a long period of time, short hairpin RNA (shRNA) was generated as a tool of the RNAi technique (21). A variety of viral and non-viral vectors can carry and express shRNA (22). Recently, studies verified successful construction of the lentivirus-shSNAIL vector, which could be infected into the HepG2 cell line to silence SNAIL (20). Adenoviral vectors harboring TRAIL were constructed by our laboratory previously (25), which all provide powerful support for our study.

In the present study, by silencing SNAIL via the utilization of RNAi in HCC cells, we investigated the effect of SNAIL deficiency for HCC cell apoptosis and the sensitization effect for TRAIL-induced apoptosis by downregulating SNAIL in HCC cells. The possible mechanism involved was also assessed.

\section{Materials and methods}

Cell line and cell culture. The human HCC HepG2, HuH-7 and HEK293 (human embryonic kidney cells) cell lines were purchased from the American Type Culture Collection (Manassas, VA, USA). SMMC7721 was purchased from the Institute of Biochemistry and Cell Biology, Chinese Academy of Sciences, Shanghai, China. The cells were maintained in a humidified condition that contained $5 \% \mathrm{CO}_{2}$ at $37^{\circ} \mathrm{C}$ and cultured in RPMI-1640 medium supplemented with $10 \%$ fetal bovine serum (FBS) and $2 \mathrm{mM}$ glutamine, followed by the addition of $100 \mathrm{U} / \mathrm{ml}$ penicillin and $100 \mu \mathrm{g} / \mathrm{ml}$ streptomycin.

Construction of lentiviral and adenoviral vectors. The most effective lentiviral vectors carrying shRNA were previously screened in our laboratory (20). Adenoviral vectors harboring TRAIL and lentiviral vectors carrying shBcl-xL, shcIAP2, shSurvivin and shRaf-1 were previously constructed by our laboratory $(20,25)$. Construction and purification of the vectors were performed as per the standard protocol. The titration of recombinant lentiviruses and adenoviruses was performed using a TCID50 assay on HEK293 cells. Recombinant lentiviral and adenoviral vectors were short for LV and Ad5, respectively. The cells were subsequently divided into the Mock, LV-shSNAIL, Ad5.TRAIL and Ad5. TRAIL + LV-shSNAIL groups.

Quantification by real-time PCR. Infected cells were collected and washed with phosphate-buffered saline (PBS). Total RNA was extracted from the cells of the target cells, and cDNA was generated with a PrimeScript ${ }^{\circledR}$ RT reagent kit (Takara, Chiga, Japan) at a total volume of $20 \mu \mathrm{l}$ according to the manufacturer's instructions. cDNA was then used in each amplification reaction. Reactions were performed using $\mathrm{SYBR}^{\circledR}$ Premix Ex Taq ${ }^{\mathrm{TM}}$ (Takara), under the following PCR conditions: denaturation at $95^{\circ} \mathrm{C}$ for $30 \mathrm{sec}$, followed by 40 cycles of annealing at $95^{\circ} \mathrm{C}$ for $5 \mathrm{sec}$ and extension at $60^{\circ} \mathrm{C}$ for $30 \mathrm{sec}$. Each sample was also subjected to melting curve analysis to confirm amplification specificity. $G A P D H$ was used as a control housekeeping gene. The expression of SNAIL was assessed by normalization of the cycle threshold $(\mathrm{Ct})$ of these genes to that of GAPDH. A Ct value was obtained from each amplification curve by using the software provided by the manufacturer (Roche, Mannheim, Germany).

Cell proliferation detection. The analysis of HCC cell viability was determined by an MTT [3-(4,5-dimethylthiazol-2-yl)-2, 5-diphenyl tetrazolium bromide] assay. HCC cells were plated in 96-well microtiter plates equally according to the density standards of $5 \times 10^{3}$ cells/well. Each well was provided suitable culture medium with the condition in $5 \% \mathrm{CO}_{2}$ at $37^{\circ} \mathrm{C}$ until grown to a high density. Each group of cells was incubated for 24-96 h following treatment with various viruses. Subsequently, $5 \mathrm{mg} / \mathrm{ml}$ MTT was added to each well and incubated for $4 \mathrm{~h}$ under the same condition. The original culture of each well was abandoned. The crystal substances produced from the cells were dissolved in $150 \mu 1$ dimethyl sulfoxide (DMSO) and agitated for $10 \mathrm{~min}$. Cell viability was assessed as optical density (OD) at a wavelength of $490 \mathrm{~nm}$ for immune monitoring by the enzyme-linked immunosorbent spot assay (Bio-Rad, Hercules, CA, USA).

Western blot analysis. Whole-cell lysates were obtained following centrifugation at $120,000 \mathrm{x}$ g for $10 \mathrm{~min}$ of the target cells of all the groups. Total proteins were separated by electrophoresis on $12 \%$ polyacrylamide and transferred to $0.45 \mu \mathrm{m}$ nitrocellulose (NC) membrane. The samples were washed three times using PBS after Ponceau S Staining kit dyeing. Bands were incubated with primary antibodies, rabbit polyclonal anti-SNAIL Santa Cruz Biotechnology, 

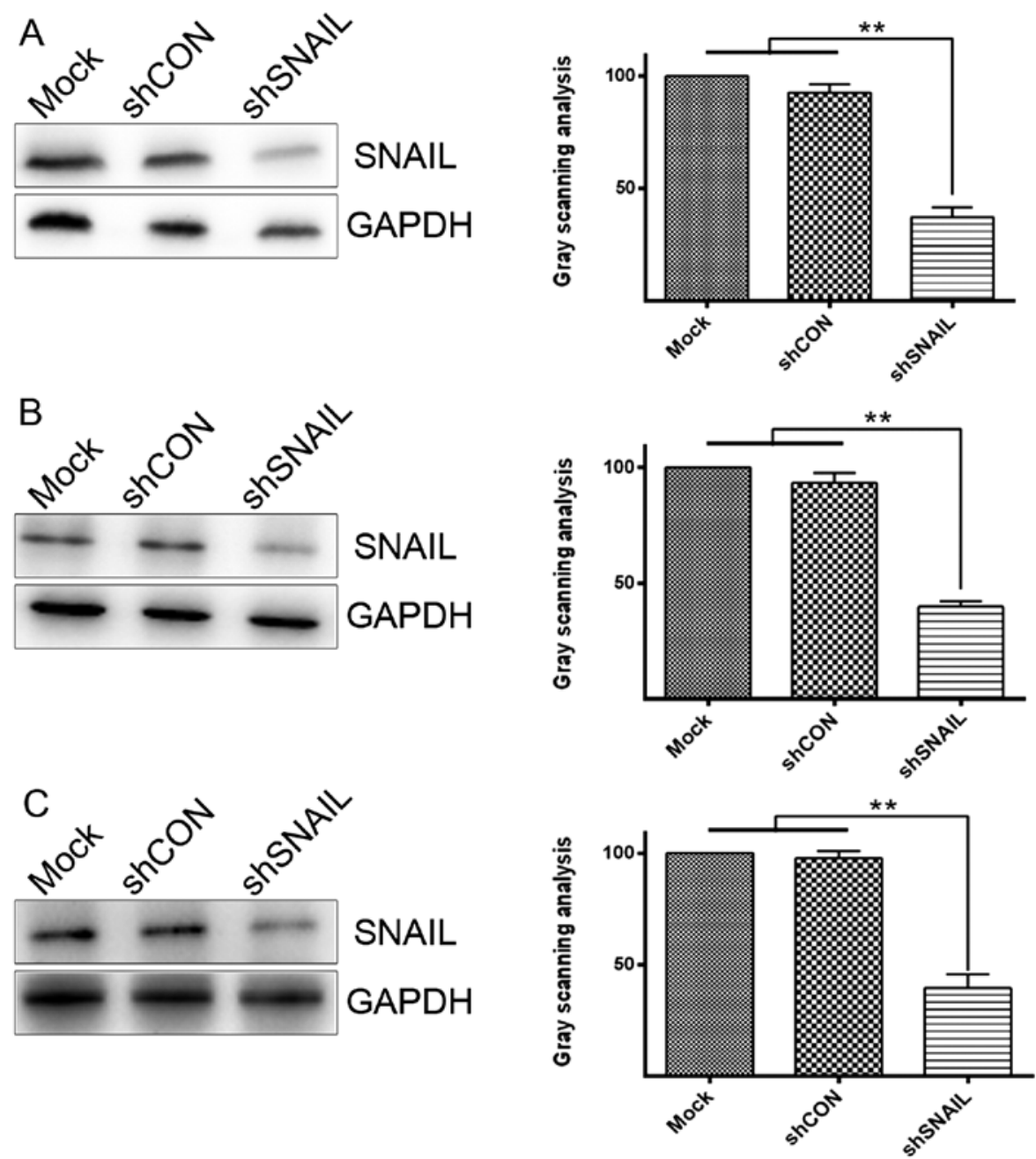

Figure 1. Characterization of LV-shSNAIL expression. SNAIL protein expression was detected prior to and after cell infection with LV- shSNAIL by western blotting using anti-SNAIL in three cell lines. Quantitative PCR was used to analyze mRNA expression level of SNAIL following infection with LV-shSNAIL in the three cell lines and control groups. The expression level of SNAIL in (A) HepG2, (B) SMMC7721 and (C) HuH-7 cells. ** P<0.05, compared with either Mock or shCON.

Inc. (Santa Cruz, CA, USA) and anti-TRAIL (Cell Signaling Technology, Danvers, MA, USA), respectively. Conjugates were combined with secondary goat anti-rabbit alkaline phosphatase antibody (Abcam, Cambridge, MA, USA), which was marked with HRP (horseradish peroxidase). GAPDH detection was performed using rabbit polyclonal anti-GAPDH and mouse monoclonal anti-GAPDH (Abcam). The fluorescent compounds were detected by ECL (Pierce, Rockford, IL, USA). At the same time, primary antibodies, rabbit polyclonal anti-p53 (Cell Signaling Technology), anti-Bcl-xL (Cell Signaling Technology), anti-cIAP2 (Abcam, Cambridge, UK), anti-survivin (Cell Signaling Technology), anti-Raf-1 (Cell Signaling Technology), respectively, were applied to the membranes and the process was repeated as above.

Hoechst test. HCC cell lines were placed into 6-well plates and incubated with recombinant lentiviruses or adenoviruses, respectively. After $48 \mathrm{~h}$, when the cells were infected, the medium was washed with PBS twice. The cells were then stained with Hoechst $33258(25 \mu \mathrm{g} / \mathrm{ml})$. Hoechst 33258 was excited by $405-\mathrm{nm}$ violet diode. The percentage of apoptotic cells was analyzed using a fluorescence microscope.
Statistical analysis. The statistical analyses were performed using the Student's t test and one-way ANOVA to determine the significance. Results were presented as mean \pm standard deviation (SD). $\mathrm{P}<0.05$ was considered statistically different.

\section{Results}

Expression of SNAIL in HCC cells. In our previous study (20), we detected the expression of SNAIL in HepG2. Based on the results, LV-shSNAIL was found to suppress the expression of SNAIL at the mRNA and protein level in HepG2 cells (20). The mRNA level was measured by employing quantitative PCR, while western blot analysis was used to determine the protein expression level. The results verified that the level of SNAIL was obviously reduced following LV-shSNAIL infection in other HCC cells (Fig. 1), which was consistent with the anterior study (20). The findings showed that the construction of LV-shSNAIL was successful in silencing SNAIL expression in various types of HCC cells.

Cell viability and apoptosis analysis in HCC cells. Recent findings have shown that, suppressing SNAIL may inhibit 


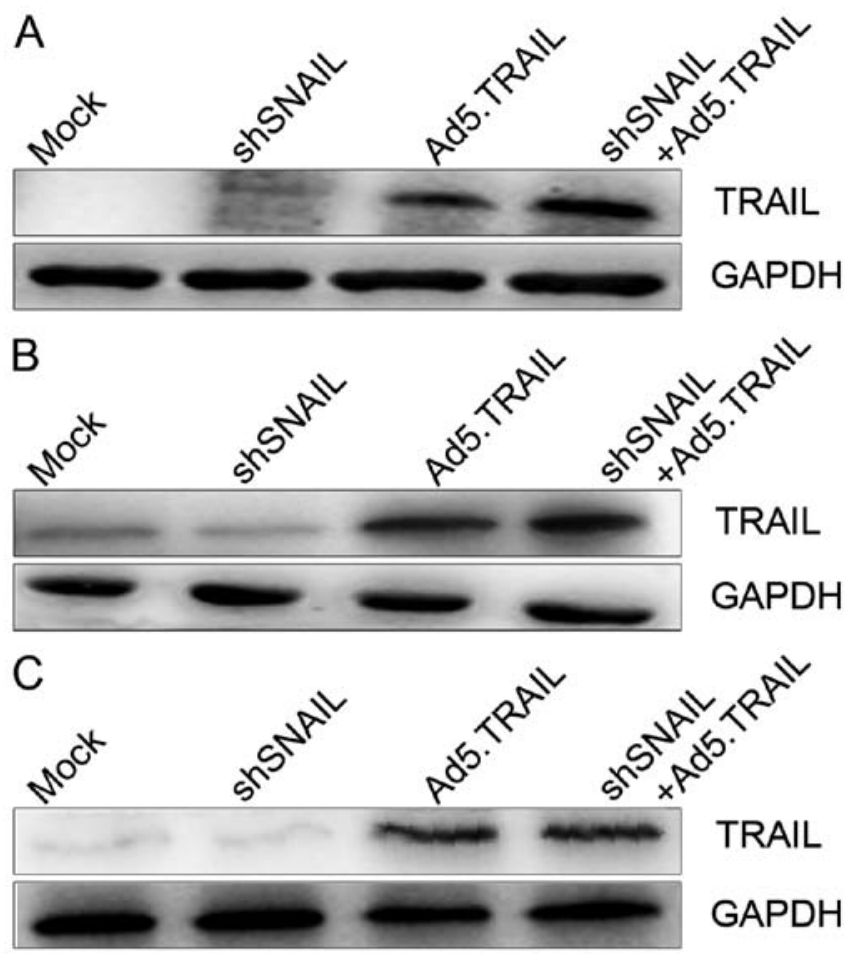

Figure 2. Effect of Ad5.TRAIL expression. TRAIL protein expression was evaluated by western blotting using anti-TRAIL in different groups (Mock, LV-shSNAIL, Ad5.TRAIL, and LV-shSNAIL + Ad5.TRAIL) of three cell lines. The expression level of TRAIL in (A) HepG2, (B) SMMC7721 and (C) HuH-7 cells.

invasion and metastasis in human HCC (26). Moreover, as described above, silencing SNAIL reduced viability in HCC cells. It is well known that the main function of TRAIL is the induction of tumor apoptosis, as identified by findings from our laboratory $(25,27)$. Therefore, we first evaluated the status of TRAIL expression in different groups of three types of HCC cells, and the level of TRAIL was detected among the cells using western blot analysis. Analysis of the results showed that TRAIL expression was markedly increased following the addition of Ad5.TRAIL + LV-shSNAIL (Fig. 2). These data showed that TRAIL was effectively expressed following Ad5.TRAIL vector infection of HCC cells, and that knockdown of SNAIL increased TRAIL expression.

We also examined the effect of SNAIL and TRAIL on HCC by assessing the state of cell proliferation. In order to study the change in HCC cell viability for response to the SNAIL silencing and TRAIL, respectively, we detected cell viability in the four groups, from the point when reagents were added until $96 \mathrm{~h}$, once every $24 \mathrm{~h}$ by MTT assay (Fig. 3). The extent of inducing apoptosis was also investigated. The samples were stained with Hoechst 33258 for $48 \mathrm{~h}$ and then analyzed by fluorescence microscopy (Fig. 4). The results revealed that silencing SNAIL or upregulated TRAIL decreased proliferation and increased apoptosis in HCC cells.

Inhibition of SNAIL enhanced TRAIL-induced apoptosis. Since TRAIL-induced apoptosis was weakened in HCC cells as compared to other TRAIL-sensitive cells, previous studies focused on apoptosis induction by acting on TRAIL for HCC cells (28). Therefore, we examined the change of
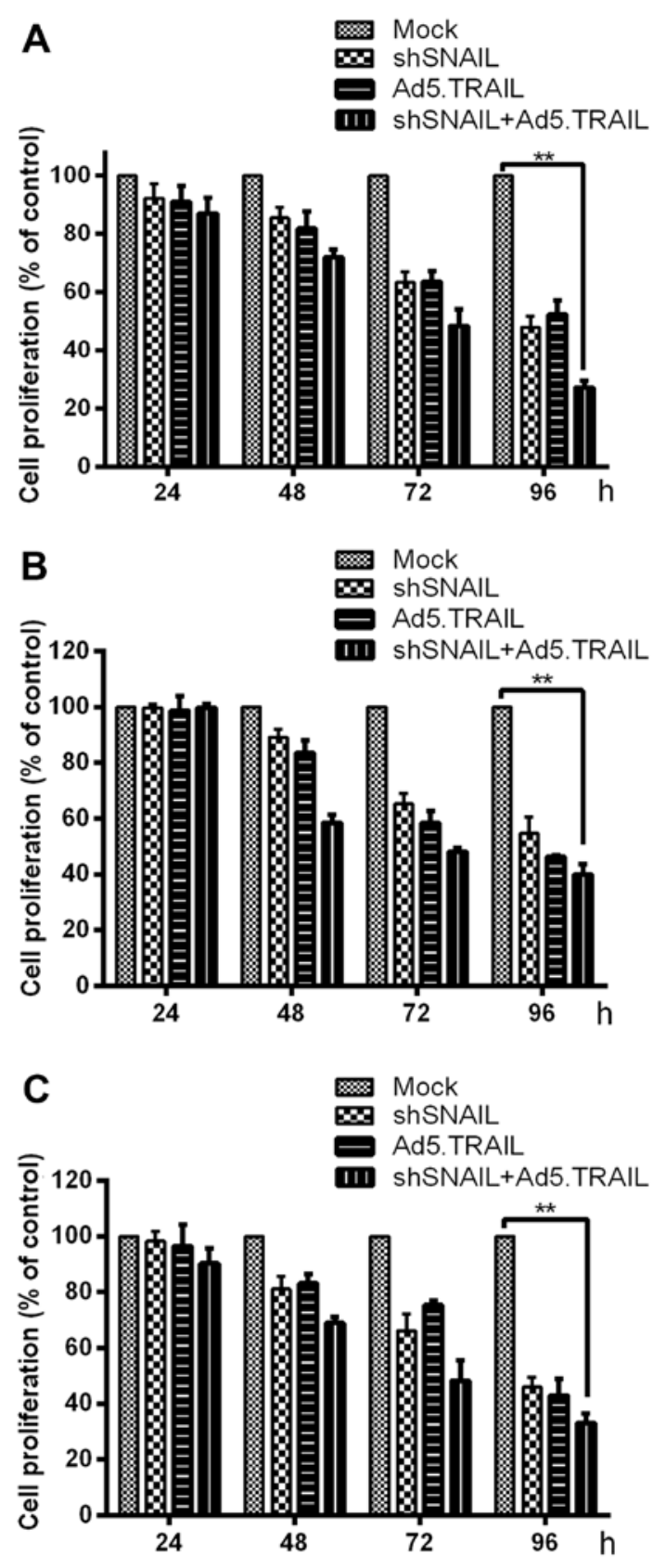

Figure 3. Effect of SNAIL knockdown and addition of TRAIL to different groups of HCC cell lines on cell proliferation. HepG2, SMMC7721 and $\mathrm{HuH}-7$ hepatocellular carcinoma cells were divided into four groups and infected with Mock, LV-shSNAIL, Ad5.TRAIL and LV-shSNAIL + Ad5.TRAIL, respectively. Cell viability was examined by MTT assay at different time points after infection. Four group reagents were added separately to (A) HepG2, (B) SMMC7721 and (C) HuH-7 cells. ${ }^{* *} \mathrm{P}<0.05$, each group compared with Mock.

TRAIL-induced apoptosis in different groups. As shown in Fig. 4, HCC cell apoptosis was highest in the group infected with LV-shSNAIL and Ad5.TRAIL compared to the other groups. This result suggested that silencing SNAIL enhanced sensitivity that TRAIL induced HCC cell apoptosis.

Knockdown of SNAIL increased TRAIL-induced apoptosis by upregulating 553 expression. Previous authors reported that p53 sensitized TRAIL-induced apoptosis in various types of cancer $(29,30)$ and blocking SNAIL activated p53, which 


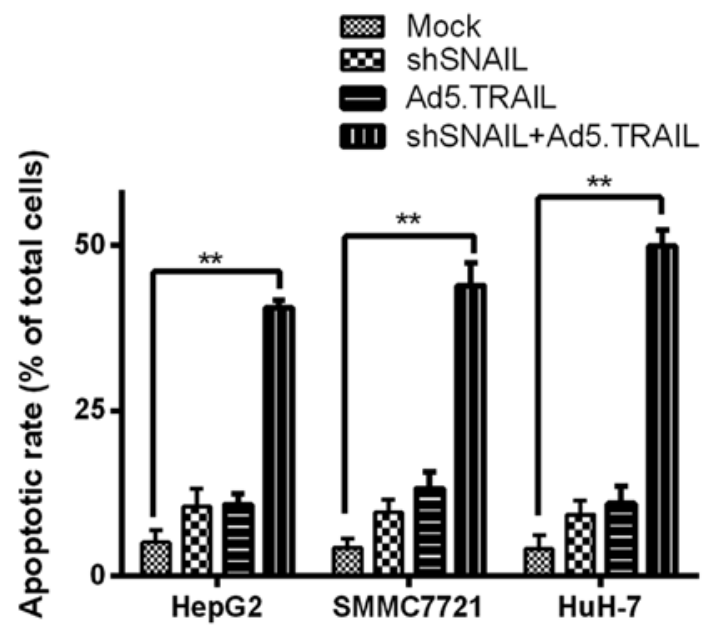

Figure 4. Knockdown of SNAIL and addition of TRAIL-induced apoptosis in the HepG2, SMMC7721 and HuH-7 cell lines. Cells were divided into four groups and incubated in Mock, LV-shSNAIL, Ad5.TRAIL and LV-shSNAIL + Ad5.TRAIL, respectively. The cells were stained with Hoechst 33258 for $48 \mathrm{~h}$ and analyzed using a fluoresence microscope. ${ }^{* * *} \mathrm{P}<0.05$, each group compared with Mock.

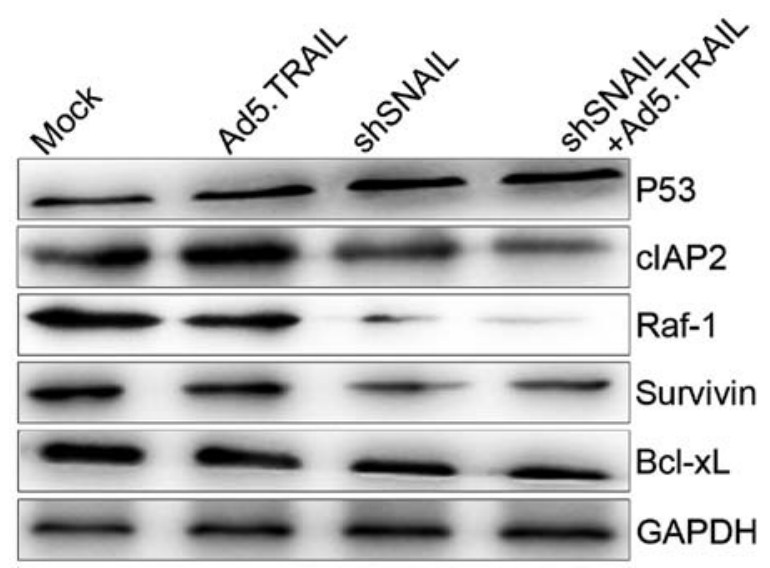

Figure 5. Expression of p53, Bcl-xL, cIAP2, survivin and Raf-1. The level was examined by western blotting in the four groups (Mock, LV-shSNAIL, Ad5.TRAIL and LV-shSNAIL + Ad5.TRAIL) of HuH-7 cells.

regulated apoptosis (31). To determine whether p53 was associated with silencing of SNAIL to increase TRAIL-induced apoptosis in HCC, we detected the expression level of p53 in different groups in $\mathrm{HuH}-7$ cells. The results revealed that $\mathrm{p} 53$ expression was highest in groups to which LV-shSNAIL was added than those where LV-shSNAIL was not added (Fig. 5).

SNAIL silencing enhanced TRAIL-induced apoptosis by affecting the NF- $\kappa B$ pathway. Resistance of HCC cells to TRAIL-induced apoptosis has been shown to be associated with Bcl-xL, cIAP2 and survivin factors, which are located in the downstream gene of the NF- $\kappa B$ pathway $(14,15,32)$. Moreover, Hall et al suggested that blocking the activation of Raf kinase inhibited NF- $\mathrm{KB}$ binding to the Mcl-1 promoter thereby enhancing the sensitization of TRAIL-mediated apoptosis in cancer cells (13). Among them, Raf-1 kinase, a member of the Raf kinase family, is an important signaling molecule acting on the downstream kinases MEK and ERK, which

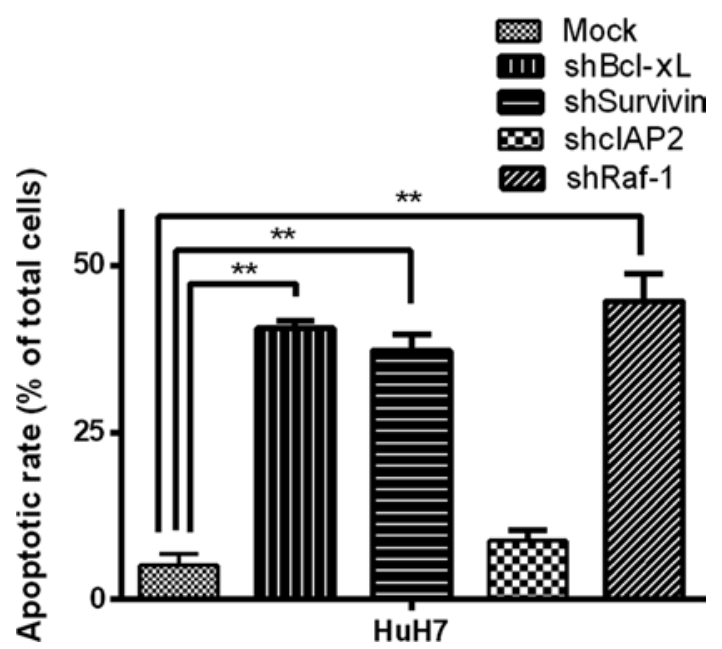

Figure 6. Effect of Bcl-xL, cIAP2, survivin and Raf-1 on TRAIL-induced apoptosis. The apoptotic rate was detected following the addition of TRAIL by Hoechst test in HuH-7 cells, which was infected by stable lentiviral-mediated shBcl-xL, shcIAP2, shSurvivin and shRaf-1. ${ }^{* *} \mathrm{P}<0.05$, compared with Mock.

pathway converges on NF- $\mathrm{B}$ activation $(13,33)$. Accordingly, we investigated the expression levels of these proteins in HCC cells to analyze the mechanism associated with the enhancement of TRAIL-induced apoptosis by SNAIL silencing. The results showed that Bcl-xL, cIAP2, survivin and Raf-1 expression were reduced following the addition of LV-shSNAIL than without its addition to HuH-7 cells, and particularly low in LV-shSNAIL + Ad5.TRAIL (Fig. 5).

In order to determine whether Bcl-xL, cIAP2, survivin and Raf-1 participated in the suppression of SNAIL to mediate HCC cell sensitivity to TRAIL-induced apoptosis, we used lentiviral vectors carrying shRNA to infect $\mathrm{HuH}-7$ for the knockdown of Bcl-xL, cIAP2, survivin and Raf-1. The effect of TRAIL-induced apoptosis of HuH-7 cells was analyzed by fluorescence microscopy following the knockdown of each gene. Infection of LV-shBcl-xL, LV-shSurvivin or LV-shRaf-1 HuH-7 cells showed obvious sensitivity to TRAIL-induced apoptosis, whereas the sensitivity of shcIAP2 was not affected (Fig. 6).

In conclusion, $\mathrm{Bcl}-\mathrm{xL}$, survivin and Raf-1 downregulation was conducive to enhancement of HCC cell sensitivity to TRAIL-induced apoptosis by silencing SNAIL. In other words, silencing of SNAIL enhanced TRAIL-induced apoptosis by affecting the NF- $\mathrm{KB}$ pathway.

\section{Discussion}

In the present study, shSNAIL carrying lentiviral and adenoviral vectors harboring TRAIL induced SNAIL silencing and enhanced TRAIL expression. The results show that HCC cell apoptosis was increased whether via silencing SNAIL or enhancing the level of TRAIL, as confirmed by the cell viability results. The results also show that the sensitivity of TRAIL-induced apoptosis was increased when SNAIL was silenced. To examine the mechanism involved, we found that p53 performed a crucial role in the sensitization of HCC cells to TRAIL by downregulating SNAIL. Other mechanisms identified showed that silencing SNAIL enhanced TRAIL-induced 
apoptosis by affecting the NF- $\mathrm{BB}$ pathway. Therefore, enhancing the sensitization of HCC cells to TRAIL is essential for the promotion of HCC cell apoptosis. Moreover, SNAIL is a potential target that may overcome resistance of HCC cells to TRAIL-induced apoptosis, rendering it an improved therapeutic strategy for HCC.

As previously mentioned, most HCC cells were resistant to apoptosis in many stimuli, which included TRAIL. However, findings of recent studies $(3,12,13,30)$ have shown that many targeted drugs alter their characteristics by acting on special targets. In the present study, we found that silencing SNAIL can increase the expression of $\mathrm{p} 53$, which alters the sensitization of HCC cells to TRAIL. On the other hand, it has been reported that p53 upregulated TRAIL death receptors $(34,35)$. Our results have shown that silencing SNAIL upregulated p53. However, whether TRAIL death receptors are correlated with p53 remains to be determined. In addition, another study has shown that adenoviral-mediated transfer of $p 53$ gene enhanced TRAIL-induced apoptosis in human HCC cells, not because of the induction of TRAIL death receptors, but due to the downregulation of cFLIP or XIAP (36), which resisted sensitization of some cell types to TRAIL-induced apoptosis $(32,37)$. Thus $p 53$ is an important gene that enhances the sensitization of HCC cells to TRAIL by downregulating cFLIP or XIAP under certain stimulation. Irrespective of the mechanism involved, p53 as a target is an important breakthrough point of HCC treatment.

$\mathrm{Bcl}-\mathrm{xL}$ as an anti-apoptosis-associated protein member of the Bcl-2 family may play a very vital role in regulating the apoptosis of HCC (38). Overexpression of Bcl-xL contributes to TRAIL resistance in cancer, including $\mathrm{HCC}(14,38-40)$. Given that Bcl-xL may be a bridge between SNAIL and TRAIL-mediated apoptosis in HCC, we further studied the relationship among them. The result showed that the expression of Bcl-xL was reduced by silencing SNAIL in HCC, and according to our experimental data, suppression of Bcl-xL by SNAIL silencing was able to increase the sensitivity of TRAIL-induced HCC cell apoptosis.

Similarly, previous studies have shown that the apoptotic inhibitors of cIAP2 and survivin are important factors in determining the apoptosis of HCC cells $(3,14)$. Thus, we investigated the expression of the two proteins. The results showed that cIAP2 and survivin were downregulated after silencing SNAIL. Additionally, we found that a decrease of survivin expression not only induced HCC cell apoptosis augment, but also enhanced the sensitivity of TRAIL-mediated apoptosis in HCC cells following the knockdown of SNAIL. However, cIAP2 did not affect TRAIL-induced apoptosis. Nevertheless, the exact mechanism on how these proteins are regulated by SNAIL silencing remains to be clarified.

A previous study demonstrated the crucial role of SNAIL in the resistance of tumor cells to TRAIL (41). As a downstream target of TRAIL, many factors acted on this role of SNAIL. Furthermore, our results show that, SNAIL is important in mediating these factors, exerting an indirect effect. SNAIL has been shown to suppress Raf-kinase inhibitor protein (RKIP) transcription and expression, which was consistent with our observation that Raf-1 protein was downregulated after SNAIL silencing, and previous findings have shown that RKIP inhibited NF- $\kappa$ B activity $(42,43)$. Another study showed that NF- $\kappa \mathrm{B}$ induces the anti-apoptotic regulator Mcl-1, which was able to cause TRAIL-resistance for cancer (13). Therefore, the potential mechanism was that silencing SNAIL could indirectly decrease Mcl-1 to mediate the sensitization of TRAIL-induced apoptosis in HCC cells by upregulating RKIP and downregulating NF- $\kappa$ B. Thus, silencing SNAIL downregulated Raf protein to influence $N F-\kappa B$ activity, thereby sensitizing HCC cells to TRAIL, which has been confirmed in our study. Nevertheless, the mechanisms involved remain to be elucidated.

In conclusion, SNAIL is a vital mediator of HCC cell sensitivity to TRAIL-induced apoptosis. Inhibition of SNAIL through shRNA carried by lentivirus is an important method that reversed HCC cell resistance to TRAIL by affecting the $\mathrm{NF}-\kappa \mathrm{B}$ pathway. Therefore, SNAIL can be a significant gene for treating $\mathrm{HCC}$ and identification of a new target to change the sensitization of TRAIL-induced apoptosis in HCC is an important therapeutic strategy.

\section{Acknowledgements}

This study was supported by grants from the National Natural Science Foundations of China (nos. 81372632 and 81402579) and the Postdoctorate Innovation Foundation of Shandong Province of China (no. 201303063).

\section{References}

1. Marquardt JU and Thorgeirsson SS: SnapShot: hepatocellular carcinoma. Cancer Cell 25: 550, 2014.

2. Yang JD and Roberts LR: Hepatocellular carcinoma: a global view. Nat Rev Gastroenterol Hepatol 7: 448-458, 2010.

3. Yamanaka Y, Shiraki K, Inoue T, Miyashita K, Fuke H, Yamaguchi Y, Yamamoto N, Ito K, Sugimoto K and Nakano T: COX-2 inhibitors sensitize human hepatocellular carcinoma cells to TRAIL-induced apoptosis. Int J Mol Med 18: 41-47, 2006.

4. Chun E and Lee KY: Bcl-2 and Bcl-xL are important for the induction of paclitaxel resistance in human hepatocellular carcinoma cells. Biochem Biophys Res Commun 315: 771-779, 2004.

5. Ogunwobi $\mathrm{OO}$ and Liu C: Hepatocyte growth factor upregulation promotes carcinogenesis and epithelial-mesenchymal transition in hepatocellular carcinoma via Akt and COX-2 pathways. Clin Exp Metastasis 28: 721-731, 2011.

6. Yang Y, Zhu J, Gou H, Cao D, Jiang M and Hou M: Clinical significance of Cox-2, Survivin and Bcl-2 expression in hepatocellular carcinoma (HCC). Med Oncol 28: 796-803, 2011.

7. Deng Q, Zhang Z, Feng X, et al: TRAIL-secreting mesenchymal stem cells promote apoptosis in heat-shock-treated liver cancer cells and inhibit tumor growth in nude mice. Gene Ther 21: 317-327, 2014.

8. Ashkenazi A and Dixit VM: Apoptosis control by death and decoy receptors. Curr Opin Cell Biol 11: 255-260, 1999.

9. Benedict CA and Ware CF: TRAIL: not just for tumors anymore? J Exp Med 209: 1903-1906, 2012.

10. Garimella SV, Gehlhaus K, Dine JL, Pitt JJ, Grandin M, Chakka S, Nau MM, Caplen NJ and Lipkowitz S: Identification of novel molecular regulators of tumor necrosis factor-related apoptosis-inducing ligand (TRAIL)-induced apoptosis in breast cancer cells by RNAi screening. Breast Cancer Res 16: R41, 2014.

11. Yamanaka T, Shiraki K, Sugimoto K, Ito T, Fujikawa K, Ito M, Takase K, Moriyama M, Nakano T and Suzuki A: Chemotherapeutic agents augment TRAIL-induced apoptosis in human hepatocellular carcinoma cell lines. Hepatology 32: 482-490, 2000.

12. Ganten TM, Koschny R, Haas TL, Sykora J, Li-Weber M, Herzer K and Walczak H: Proteasome inhibition sensitizes hepatocellular carcinoma cells, but not human hepatocytes, to TRAIL. Hepatology 42: 588-597, 2005.

13. Hall MA and Cleveland JL: Clearing the TRAIL for cancer therapy. Cancer Cell 12: 4-6, 2007. 
14. Omar HA, Arafa el-SA, Maghrabi IA and Weng JR: Sensitization of hepatocellular carcinoma cells to Apo2L/TRAIL by a novel Akt/NF- $\kappa \mathrm{B}$ signalling inhibitor. Basic Clin Pharmacol Toxicol 114: 464-471, 2014.

15. Refaat A, Abd-Rabou A and Reda A: TRAIL combinations: The new 'trail' for cancer therapy (Review). Oncol Lett 7: 1327-1332, 2014.

16. Peinado H, Olmeda D and Cano A: Snail, Zeb and bHLH factors in tumour progression: an alliance against the epithelial phenotype? Nat Rev Cancer 7: 415-428, 2007.

17. Pioli PD and Weis JH: Snail transcription factors in hematopoietic cell development: a model of functional redundancy. Exp Hematol 42: 425-430, 2014.

18. Kudo-Saito C, Shirako H, Takeuchi T and Kawakami Y: Cancer metastasis is accelerated through immunosuppression during Snail-induced EMT of cancer cells. Cancer Cell 15: 195-206, 2009.

19. Miyoshi A, Kitajima Y, Kido S, Shimonishi T, Matsuyama S, Kitahara K and Miyazaki K: Snail accelerates cancer invasion by upregulating MMP expression and is associated with poor prognosis of hepatocellular carcinoma. Br J Cancer 92: 252-258, 2005.

20. Liu J, Jiang G, Liu S, Liu Z, Pan H, Yao R and Liang J: Lentivirusdelivered short hairpin RNA targeting SNAIL inhibits HepG2 cell growth. Oncol Rep 30: 1483-1487, 2013.

21. Fellmann C and Lowe SW: Stable RNA interference rules for silencing. Nat Cell Biol 16: 10-18, 2014.

22. Takakura Y: Towards therapeutic application of RNA-mediated gene regulation. Preface. Adv Drug Deliv Rev 61: 667, 2009.

23. Djuranovic S, Nahvi A and Green R: A parsimonious model for gene regulation by miRNAs. Science 331: 550-553, 2011.

24. Jinek M and Doudna JA: A three-dimensional view of the molecular machinery of RNA interference. Nature 457: 405-412, 2009.

25. Liu X, Wang J, Wang H, Liu S, Liang Y, Lv Z, Zhou Q and Ding W: Combination of Ad-sTRAIL with the chemotherapeutic drug cisplatin synergistically enhances their pro-apoptotic ability in human breast cancer cells. Oncol Rep 30: 1913-1919, 2013.

26. Chen D, Zheng X, Jiao X, Gao Y, Zhang $\mathrm{K}$ and Liang J: Transcriptional repressor snail and metastasis in hepatocellular carcinoma. Hepatogastroenterology 59: 1359-1365, 2012.

27. Smyth MJ, Takeda K, Hayakawa Y, Peschon JJ, van den Brink MR and Yagita H: Nature's TRAIL - on a path to cancer immunotherapy. Immunity 18: 1-6, 2003.

28. Abou El Naga RN, Azab SS, El-Demerdash E, Shaarawy S, El-Merzabani M and Ammar el-SM: Sensitization of TRAILinduced apoptosis in human hepatocellular carcinoma HepG2 cells by phytochemicals. Life Sci 92: 555-561, 2013.

29. Liu X, Qiu F, Liu Z, Lan Y, Wang K, Zhou PK and Wang Y: Urokinase-type plasminogen activator receptor regulates apoptotic sensitivity of colon cancer HCT116 cell line to TRAIL via JNK-p53 pathway. Apoptosis 19: 1532-1544, 2014.

30. Zhao J, Lu Y and Shen HM: Targeting p53 as a therapeutic strategy in sensitizing TRAIL-induced apoptosis in cancer cells Cancer Lett 314: 8-23, 2012.
31. Lee SH and Park BJ: p53 activation by blocking Snail: a novel pharmacological strategy for cancer. Curr Pharm Des 17: 610-617, 2011.

32. Finlay D, Vamos M, Gonzalez-Lopez M, et al: Small-molecule IAP antagonists sensitize cancer cells to TRAIL-induced apoptosis: roles of XIAP and cIAPs. Mol Cancer Ther 13: 5-15, 2014.

33. Dougherty MK, Müller J, Ritt DA, Zhou M, Zhou XZ, Copelan TD, Conrads TP, Veenstra TD, Lu KP and Morrison DK: Regulation of Raf-1 by direct feedback phosphorylation 17 : 215-224, 2005

34. Akram KM, Lomas NJ, Forsyth NR and Spiteri MA: Alveolar epithelial cells in idiopathic pulmonary fibrosis display upregulation of TRAIL, DR4 and DR5 expression with simultaneous preferential over-expression of pro-apoptotic marker p53. Int J Clin Exp Pathol 7: 552-564, 2014.

35. Yeh $\mathrm{CH}$, Yang YY, Huang YF, Chow KC and Chen MF: Induction of apoptosis in human Hep3B hepatoma cells by norcantharidin through a p53 independent pathway via TRAIL/DR5 signal transduction. Chin J Integr Med 18: 676-682, 2012.

36. Inoue H, Shiraki K, Murata K, et al: Adenoviral-mediated transfer of p53 gene enhances TRAIL-induced apoptosis in human hepatocellular carcinoma cells. Int J Mol Med 14: 271-275, 2004

37. Cantarella G, Di Benedetto G, Ribatti D, Saccani-Jotti G and Bernardini R: Involvement of caspase 8 and c-FLIPL in the proangiogenic effects of the tumour necrosis factor-related apoptosis-inducing ligand (TRAIL). FEBS J 281: 1505-1513, 2014.

38. Guo XZ, Shao XD, Liu MP, Xu JH, Ren LN, Zhao JJ, Li HY and Wang D: Effect of bax, bcl-2 and bcl-xL on regulating apoptosis in tissues of normal liver and hepatocellular carcinoma. World $\mathbf{J}$ Gastroenterol 8: 1059-1062, 2002.

39. Bansal H, Seifert T, Bachier C, Rao M, Tomlinson G, Iyer SP and Bansal S: The transcription factor Wilms tumor 1 confers resistance in myeloid leukemia cells against the proapoptotic therapeutic agent TRAIL (tumor necrosis factor alpha-related apoptosis-inducing ligand) by regulating the antiapoptotic protein Bcl-xL. J Biol Chem 287: 32875-32880, 2012.

40. Koehler BC, Urbanik T, Vick B, Boger RJ, Heeger S, Galle PR, Schuchmann M and Schulze-Bergkamen H: TRAIL-induced apoptosis of hepatocellular carcinoma cells is augmented by targeted therapies. World J Gastroenterol 15: 5924-5935, 2009.

41. Kaler P, Galea V, Augenlicht L and Klampfer L: Tumor associated macrophages protect colon cancer cells from TRAIL-induced apoptosis through IL-1beta-dependent stabilization of Snail in tumor cells. PLoS One 5: e11700, 2010.

42. Baritaki S and Bonavida B: Viral infection and cancer: the NF-kappaB/Snail/RKIP loop regulates target cell sensitivity to apoptosis by cytotoxic lymphocytes. Crit Rev Immunol 30: 31-46, 2010.

43. Wu K and Bonavida B: The activated NF-kappaB-Snail-RKIP circuitry in cancer regulates both the metastatic cascade and resistance to apoptosis by cytotoxic drugs. Crit Rev Immunol 29: 241-254, 2009 